myopathy in CKD (12). If there is truth to this model, one may not want to obliterate FGF23 but rather direct FGF23 back to its more physiologic actions, perhaps by restoring Klotho levels.

\section{Looking ahead}

In sum, one should not view the study of Shalhoub and coworkers as a nihilistic "show stopper" in designing FGF23-related therapeutics in CKD. Instead, it should alert the field of the need to abandon the overly simplistic FGF23 toxicity model. Efforts should be directed toward defining the roles of FGF23 in physiology and CKD. What is actually driving up the FGF23 levels in CKD? What are the optimal levels in various stages of CKD for its different roles? What are the ways to enhance endogenous on-target signaling as adaptive reactions and decrease the off-target effects? Instead of finding the singular magic bullet, perhaps derangements of mineral metabolism need to be attacked at multiple loci. FGF23 is likely both friend and foe in CKD, and much knowledge and wisdom needs to be secured before we can move toward therapeutic applications.

\section{Acknowledgments}

The author is supported by the NIH, the Simmons Family Foundation, the Charles and Jane Pak Foundation, and an investigator-initiated GRIP research grant from Genzyme Corporation.

Address correspondence to: Orson W. Moe, Departments of Internal Medicine and Physiology, University of Texas Southwestern Medical Center, 5323 Harry Hines Blvd., Dallas, Texas 75390, USA. Phone: 214.648.7993; Fax: 214.648.2071; E-mail: Orson.Moe@utsouthwestern.edu.

1. [No authors listed]. Strategies for influencing outcomes in pre-ESRD and ESRD patients: a special conference. 1998. Proceedings. Am J Kidney Dis. 1998;32(6 suppl 4):S1-S193.

2. Bernard C. Introduction a L'etude de la Medecine Experimentale. Paris, France: Éditions GarnierFlammarion; 1865.

3. D'Apolito M, et al. Urea-induced ROS generation causes insulin resistance in mice with chronic renal failure. JClin Invest. 2010;120(1):203-213.

4. European Uremic Toxin (EUTox) Work Group of the ESAO and ERA-EDTA. EUTox Web site. www.uremic-toxins.org. Accessed May 23, 2012

5. Singh AK. What is causing the mortality in treating the anemia of chronic kidney disease: erythropoietin dose or hemoglobin level? Curr Opin Nephrol Hypertens. 2010;19(5):420-424.

6. The National Kidney Foundation Kidney Disease Outcomes Quality Initiative (NKF KDOQI). National Kidney Foundation Web site. http:// www.kidney.org/professionals/KDOQI/guidelines_commentaries.cfm. Accessed May 23, 2012.

7. Clinical practice guidelines. Kidney Disease: Improving Global Outcomes (KDIGO) Web site. http://www.kdigo.org/clinical_practice_guidelines/index.php. Accessed May 23, 2012.
8. ADHR-Consortium. Autosomal dominant hypophosphataemic rickets is associated with mutations in FGF23. Nat Genet. 2000;26(3):345-348

9. Shimada $\mathrm{T}$, et al. Cloning and characterization of FGF23 as a causative factor of tumor-induced osteomalacia. Proc Natl Acad Sci U S A. 2001; 98(11):6500-6505.

10. Martin A, David V, Quarles LD. Regulation and function of the FGF23/klotho endocrine pathways. Physiol Rev. 2012;92(1):131-155.

11. Isakova T. Fibroblast growth factor 23 and adverse clinical outcomes in chronic kidney disease. Curr Opin Nephrol Hypertens. 2012;21(3):334-340.

12. Faul C, et al. FGF23 induces left ventricular hypertrophy. J Clin Invest. 2011;121(11):4393-4408.

13. Goetz R, et al. Isolated C-terminal tail of FGF23 alleviates hypophosphatemia by inhibiting FGF23FGFR-Klotho complex formation. Proc Natl Acad Sci US A. 2010;107(1):407-412

14. Razzaque MS. Therapeutic potential of klothoFGF23 fusion polypeptides: WO2009095372. Expert Opin Ther Pat. 2010;20(7):981-985.

15. Hasegawa $\mathrm{H}$, et al. Direct evidence for a causative role of FGF23 in the abnormal renal phosphate handling and vitamin D metabolism in rats with early-stage chronic kidney disease. Kidney Int. 2010;78(10):975-980

16. Yamazaki Y, et al. Anti-FGF23 neutralizing antibodies show the physiological role and structural features of FGF23. J Bone Miner Res. 2008; 23(9):1509-1518.

17. Shalhoub V, et al. FGF23 neutralization improves chronic kidney disease-associated hyperparathyroidism yet increases mortality. J Clin Invest. 2012; 122(7):2543-2553.

18. Zoccali C, et al. Phosphate may promote CKD progression and attenuate renoprotective effect of ACE inhibition. J Am Soc Nephrol. 2011;22(10):1923-1930.

19. Hu MC, et al. Klotho deficiency and vascular calcification in chronic kidney disease. J Am Soc Nephrol. 2011;22(1):124-136

\title{
Pharmacogenomics: mapping monogenic mutations to direct therapy
}

\author{
Palmer Taylor \\ Department of Pharmacology, Skaggs School of Pharmacy and Pharmaceutical Sciences, UCSD, La Jolla, California, USA.
}

\begin{abstract}
The molecular mapping of mutations that underlie congenital disorders of monogenic origin can result in both a broader understanding of the molecular basis of the disorder and novel therapeutic insights. Indeed, genotyping patients and then replicating the behavior of the mutant gene products in welldefined biochemical or electrophysiological systems will allow tailoring of therapy to be mutation- and protein sequence-dependent. In this issue of the JCI, Shen and colleagues describe such an approach that identified novel mutations in the $\alpha$ subunit of the nicotinic receptor linked to myasthenia gravis.
\end{abstract}

In this issue of the JCI, Shen and colleagues detail the discovery of novel mutations

Conflict of interest: The author holds stock in Merck, Pfizer, Sequenom, Qualcom, Lilly, and Abbott and receives research support from Targacept.

Citation for this article: J Clin Invest. 2012; 122(7):2356-2358. doi:10.1172/JCI64409. linked to myasthenia gravis (1), a disease characterized by skeletal muscle weakness, typically originating at the neuromuscular junction. This investigation is one of a series from the Engel and Sine groups at the Mayo Clinic that are unraveling the genetic basis of the disease. Around 15\% of myasthenia gravis disorders are not of the autoimmune type and are linked to mutations in genes encoding proteins in the neuromuscular junction. These can range from the enzymes, transporters, and docking proteins found presynaptically in the motor neuron that control synthesis and release of acetylcholine, to cholinesterases of postsynaptic origin that catalyze the rapid hydrolysis of the released acetylcholine neurotransmitter. Between the synthesis and release of acetylcholine and the destruction of the neurotransmitter lies the nicotinic receptor, which has the capacity to recognize acetylcholine and respond rapidly to neurotransmitter binding through channel gating. This initiates a depolarization and postsynaptic potential, 


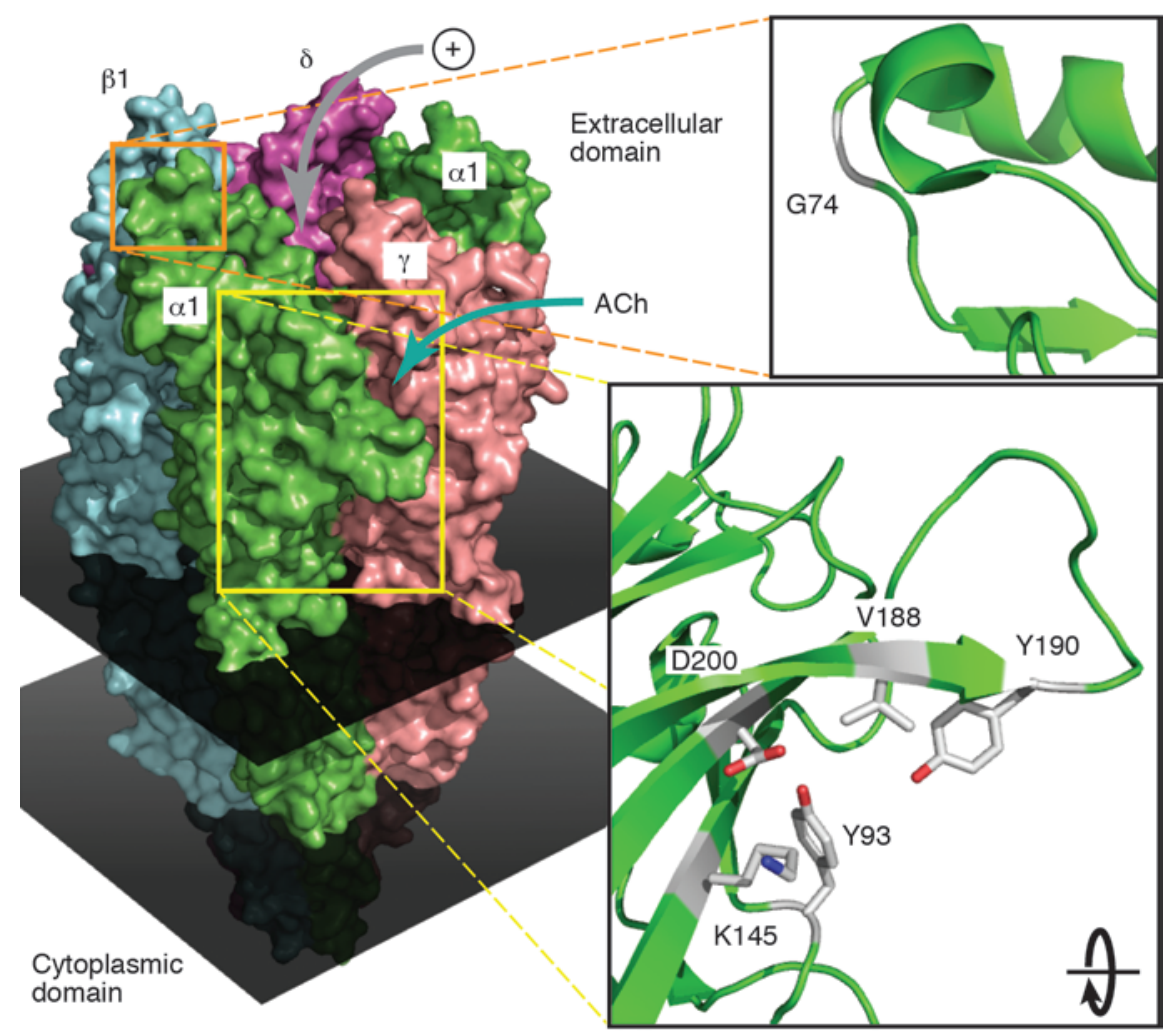

Figure 1

Space-filling structure of the muscle nicotinic acetylcholine receptor (m-nAChR). Shown is a representation of the muscle nAChR from the Torpedo sp. electric organ based on the electron microscopy reconstructions of Unwin (13). The pentameric structure in Torpedo and mammalian skeletal muscle consists of two copies of an $\alpha$ subunit, one $\beta$, one $\delta$, and one $\gamma$ or $\varepsilon$, depending on whether the muscle receptor is innervated. The portion between the two parallel dark gray sheets represents the transmembrane span. Acetylcholine binds cooperatively at the $\alpha-\gamma$ and $\alpha-\delta$ subunit interfaces, as shown by the radial entry of the blue arrow, causing the channel gate, located in the transmembrane span, to open. lon entry is shown by the gray arrow leading into a central vestibule of the channel surrounded by the five subunits. The structure is based on ref. 14 (Protein Data Bank [PDB] 2BG9). The insets show ribbon diagrams of portions of the $\alpha$ subunit where the two mutations, G74C and V188M, reside. Also shown in the lower inset in white, with red denoting oxygen and blue nitrogen, are the aromatic side chains $Y 190$ and $Y 93$ and charged side chains D200 and K145, forming the proposed tetrad of interacting side chains involved in activation (1). The diagrams are based on a crystal structure of the $\alpha 1$ subunit from mouse complexed with the snake toxin $\alpha$-bungarotoxin (ref. 15; PDB 2QC1). The toxin structure has been removed for clarity.

triggering an action potential that, in turn, initiates muscle contraction (2). The pentameric nicotinic receptor in innervated muscle, composed of two copies of the $\alpha$ subunit and one copy each of the $\beta, \varepsilon(\gamma)$, and $\delta$ subunits, is the locus of the majority of identified mutations in the neuromuscular junction giving rise to congenital myasthenia (3).

Engel and Sine have been masters in finding and characterizing congenital mutations in various genes by analyzing patients identified on the basis of unusual patterns in their myasthenic phenotype (4-6). Hence, from initial referrals based on neu- romuscular phenotype, Engel and Sine have employed multiple approaches, including DNA sequencing and subjecting muscle biopsies to biochemical and electrophysiological analysis, to categorize and annotate these congenital monogenic disorders.

A review of previously described disease- or disorder-linked mutations at the neuromuscular junction reveals a great variety of single-gene mutations, and Sine and Engel have systematically categorized them with respect to neuromuscular function. What emerged from this effort was the finding

\section{Monogenic myasthenia gravis}

that the mutations affecting the nicotinic receptor may either influence biosynthesis and trafficking of the nascent protein or the gating kinetics of the functional receptor in the synapse. Altered gating kinetics can be divided into fast- and slow-channel syndromes distinguished by the kinetic constants for channel opening and closing. An extensive array of mutations of low abundance have been found in the $\alpha$, $\beta, \varepsilon$, and $\delta$ subunits of the human muscle receptor and have been mapped to distinct regions in the quaternary structure (4-6).

Deficiency of nicotinic receptor expression typically arises from mutations in the promoter region or open reading frame of the $\varepsilon$ subunit. With diminished subunit expression or compensatory expression of the $\gamma$ subunit, the margin of safety for neuromuscular transmission (which requires sufficient functional receptors to trigger end plate and action potentials) is compromised (5). Acetylcholinesterase inhibitors that prolong acetylcholine lifetimes in the synapse and likely promote its lateral diffusion and 3,4-diaminopyridine, which enhances presynaptic release, offer favorable therapeutic options for diminished expression.

In slow-channel syndromes, the association of acetylcholine with the receptor and/or the efficiency of channel opening is enhanced, giving rise to prolonged and often biphasic miniature end plate currents. The slow-channel syndrome is further compromised by cholinesterase inhibitors, but allosteric agents that enter an open channel such as fluoxetine, quinine, or its isomer quinidine can ameliorate the condition (4-6).

In the fast-channel syndromes, affinity for acetylcholine is diminished and gating to the open channel is reduced, affording very short open channel lifetimes and enhanced rates of decay of miniature end plate currents. Patients respond well to 3,4-diaminopyrdine, which promotes the release of presynaptic acetylcholine or to cholinesterase inhibitors, acting as described above (4-6).

\section{Novel nicotinic receptor mutations}

In the current study, Shen and colleagues describe two mutations in the $\alpha$ subunit of the nicotinic receptor in a patient who has had myasthenic syndrome since birth (1). These amino acid substitutions are G74C, in the main immunogenic region located apically in the extracellular domain, a region responsible for much of the autoimmune myasthenia; and V188M, located just $\mathrm{N}$-terminal to the C-loop. The first mutation appears to result in reduced expression 
levels of the $\alpha$ subunit and assembled receptor. As yet, it is unclear whether this could reflect a limitation in folding of the nascent protein, aberrant disulfide bond formation, trafficking of the protein, or perhaps a subacute immune response affecting steadystate expression levels.

The V188M mutation in the C-loop is largely responsible for the phenotype and appears to be functionally linked to two residues in the $\beta$ strand - Tyr190 and Asp200that lead into the M1 transmembrane span (Figure 1). The V188M mutation is structurally conservative, not altering charge, but instead enlarging side chain dimensions. The authors propose that inter-residue coupling in the C-loop among $\alpha V 188 \mathrm{M}$, $\alpha$ Tyr190, and $\alpha$ Asp200, along with $\alpha$ Lys145, forms an interacting tetrad. The tetrad mediates a conformational change upon agonist binding that extends from the C-loop to the constriction point in the transmembrane channel (Figure 1). Channel opening rates and gating efficiency are reduced some 70to 80 -fold by this mutation (1).

\section{The pharmacogenomics approach in monogenic disorders}

Accordingly, we are left with the laborious, but attractive, prospect of molecular mapping of the mutations and replicating the behavior of the gene products in well-defined biochemical or electrophysiological systems. This will allow tailoring of therapy that might be mutation- and protein sequence-dependent. Indeed, the experimental endeavor involves translation from patient phenotype, to muscle biopsy, and finally to molecular analysis in deconstructed systems. Improved function is achieved through enhanced protein expression via folding and trafficking or compensating for aberrant sub-millisecond channel opening and closing events resulting from the mutations.

There are several well-characterized monogenic mutations reflected in proteins that directly impact neuromuscular signaling, for which therapeutic progress has emerged only after a thorough characterization of phenotype and genotype. These include butyrylcholinesterase deficiency, a disorder in which seemingly normal patients receiving succinylcholine during surgical procedures develop apnea and require resuscitation (7). The characterization the multiple polymorphisms of the single gene linked to this disorder opened the door to the field of pharmacogenomics. Similarly, in malignant hyperthermia, phenotype-genotype analysis has led to the understanding that a vast majority of patients have mutations in the gene encoding the ryanodine receptor of skeletal muscle (RYR1) or in a gene encoding the associated calcium channel subunits (8). The mutations lead to a gain in function resulting in excessive calcium release, muscle ATP hydrolysis, and heat generation. This can be treated therapeutically with dantrolene, which, acting at the level of the sarcoplasmic reticulum, antagonizes this cascade of metabolic, heat-generating events.

New therapeutic avenues have become apparent for other monogenic disorders of greater frequency in human populations, such as cystic fibrosis, where we now have the prospect of using small molecules to serve as chaperones to enhance expression and facilitate trafficking of the mutant protein from the endoplasmic reticulum to its transmembrane location (9-13). Here again, individual mutations in the affected protein, the cystic fibrosis transmembrane conductance regulator (CFTR), require identification before treatment, for it appears that CFTR potentiators, by an as yet not fully understood mechanism, show selective efficacy in rectifying trafficking or function of particular mutant CFTR proteins (9). For example, the lead candidate in the potentiator molecule class, VX-770, appears efficacious for only the G551D mutation in CFTR (9), which is present in less than $5 \%$ of the CF population (10). A congeneric compound shows wider application for treatment of patients harboring the common CFTR mutation F508del (11). This mutation is responsible for compromised transport function in several secretory tissues of cystic fibrosis patients (10). Hence, despite the fact the condition is monogenic, phenotype as well as responsiveness to therapy appear mutation dependent, a theme recapitulated in the current study of nicotinic receptor mutations. As illustrated in the Shen et al. study (1), when new mutations are uncovered and successful therapy is mutation based, we can achieve an intimate understanding of the mechanisms of the functioning receptor and its expression.

\section{Conclusions}

Often pharmacogenomics is subdivided into the study of mutations affecting drug disposition through drug metabolism and transport (mutations that affect pharmacokinetics) and those that alter drug action at the target site (mutations affecting pharmacodynamics). Another consideration in contemporary pharmacogenomics relates to whether the mutation(s) influencing therapeutic outcomes in treatment or prevention of disorders have an underlying monogenic or polygenic basis. As the study by Shen et al. shows, drilling deep to identify the mutation (in this case two mutations) in the gene and ascertaining their effects on structure and function of the gene product provide the pathway to diagnosis, therapy, and patient management in congenital myasthenic syndromes (1). Indeed this approach sets a paradigm for diagnosis and treatment of other monogenic congenital disorders.

\section{Acknowledgments}

I thank Akos Nemecz for the production of Figure 1.

Address correspondence to: Palmer Taylor, Pharmaceutical Sciences Bldg 0657, University of California, San Diego, La Jolla, California 92093, USA. Phone: 858.534.1366; Fax: 858.822.5591; E-mail: pwtaylor@ucsd.edu.

1. Shen X-M, Brengman JM, Sine SM, Engel AG. Myasthenic syndrome AChR $\alpha$ C-loop mutant disrupts initiation of channel gating. J Clin Invest. 2012; 122(7):2613-2621.

2. Karlin A. Emerging structure of the nicotinic acetylcholine receptors. Nat Rev Neurosci. 2002;3(2):102-114.

3. Changeux JP. Allosteric receptors from electric organs to cognition. Annu Rev Pharmacol Toxicol. 2010;50:1-38.

4. Engel AG. Current status of the congenital myasthenic syndromes. Neuromuscul Disord. 2012;22(2):99-111.

5. Engel AG, Ohno K, Sine SM. Sleuthing molecular targets for neurological diseases at the neuromuscular junction. Nat Rev Neurosci. 2003;4(5):339-352.

6. Sine SM, Engel AG. Recent advances in Cys loop receptor structure and function. Nature. 2006; 440(7083):448-455

7. Kalow W. Pharmacogenetics: a historical perspective. In: Pharmacogenomics: Applications to Patient Care. 2nd ed. Lenexa, Kansas, USA: American College of Clinical Pharmacy; 2004:251-270.

8. Rosenberg H, Davis M, James D, Pollock N, Stowell K. Malignant hyperthermia. Orphanet J Rare Dis. 2007;2:21.

9. Accurso FJ, et al. Effect of VX-770 in persons with cystic fibrosis and the G551D CFTR mutation, N Engl J Med. 2010;363(21):1991-2003.

10. Bobadilla JL, Macek M Jr, Fine JP, Farrell PM. Cystic fibrosis: a worldwide analysis of CFTR mutationscorrelation with incidence data and application to screening. Hum Mutat. 2002;19(6):575-606.

11. Van Goor F, et al. Correction of the F508del-CFTR protein processing defect in vitro by the investigational drug VX-809. Proc Natl Acad Sci. 2011; 108(46):18843-18848.

12. Becq F. Cystic fibrosis transmembrane conductance regulator modulators for personalized drug treatment in cystic fibrosis. Drugs. 2010; $70(3): 241-259$

13. Mendoza JL, et al. Requirements for efficient correction of $\Delta$ F508 CFTR revealed by analyses of evolved sequences. Cell. 2012;148(1-2):164-174.

14. Unwin N. Refined structure of the nicotinic acetylcholine receptor at $4 \mathrm{~A}$ resolution. J Mol Biol. 2005;346(4):967-989.

15. Dellisanti CD, Yao Y, Stroud JC, Wang ZZ, Chen L. Crystal structure of the extracellular domain of $\mathrm{nAChR} \alpha 1$ bound to $\alpha$-bungarotoxin at $1.94 \mathrm{~A}$ resolution. Nat Neurosci. 2007;10(8):953-962. 